\title{
Unmanned aerial vehicle quadcopter: a review
}

\begin{abstract}
Aerial vehicles of the multicopter type are vertical take-off and landing (VTOL) vehicles, which have various civil and military applications. Unmanned aerial vehicles (UAVs) are a prolific category of robotics in which additional research is required. The objective of this work is to comprehensively review the area of UAV multicopters, with a focus on quadcopter types, with respect to information summarized from 123 published research works, which have been divided into primary sections for consideration. This paper describes the types of unmanned vehicles, the quadcopter flying mechanism, quadcopter control techniques, propeller modeling, multicopter control boards, multicopter sensors and the various practical applications of multicopters. Finally, we suggest a quadcopter application that has not previously been described in the related literature, and we discuss the searching pattern used when flying such a robot.
\end{abstract}

Keyword: Landfill; Pattern; Quadcopter; Risk; Sniffer; UAV 